\title{
SORODIAGNOSTICO DO CANCER (*)
}

\author{
PROF. JAYME REGALO PEREIRA \\ (Catedrático de Farmacolugia da Faculdade de Medicina da Universidade \\ de São Paulo
}

Nunca, como atualmente, o problema do cancer esteve tão focalizado, merecendo a atenção dos clinicos e homens de laboratorio. $E$ apesar do enorme acervo de trabalhos realizados para um melhor cónhecimento deste problema, o que se pode apurar na conta de credito dos investigadores é excessivamente pouco, comparando-se com o que continúa registrado na conta de debito. Mais e mais o cancer invade as estatisticas nosologicas; seja porque prolifera a entidade morbida, seja porque novos meios propedeuticos podem hoje revelar com mais segurança os casos diagnosticados cqmo tal. O fato é que o can ${ }^{\perp}$ cer como uma grande sombra negra se projeta sobre a humanidade e por todos os lados arregimentam-se médicos e cientistas, barricadas se levantam, todos os meios de defesa são procurados no afan de enfrentar a onda avassaladora que promete destruir o genero humano.

Á gentileza de meus amigos, os estudantes de medicina de nossa Faculdade, devo eu a honra de ocupar neste momento a atenção dos Senhores. Muitos dos que aqui se acham, si não mesmo tòdos, espantar-se-ão ao me verem nesta tribuna, a tratar de uma questão médica para a qual jamais se voltaram minhas preocupações cientificas e que somente de muito longe pode relacionar-se com minha especialidade - a Farmacologia. Não é, porem, minha intenção esclarecer qualquer duvida relativa ao problema do cancer. Proponho-me tão somente trazer ao vossa conhecimento os resultados de pesquizas alheias que me pareceram assaz interessantes, capazes de estimularem novas pesquizas por parte dos que, entre nós, possam ser arregimentados para a campanha contra o cancer. Faço-me apenas o porta vóz de outros pesquizadores com os quais tomei contáto em minha recente viagem á Alemanha e que de alguns anos a esta parte veem estudando com paciencia uma das faces do problema, qual a do diagnostico dessa molestia.

Tão relevantes se me afiguram esses resultados, que sua significação não se limita apenas ao campo do diagnostico, projetando ainda fortes luzes sobre os terrenos da etiologia e até mesmo da terapeutica.

(*) Conferencia realizada em sessão solene da Liga de Combate ao Cancer, a 21-6-1940. 
Recapitulemos os fatos.

Convidado pelo Instituto Ibero-Americano para uma viagem de estudos á Alemanha, no ano passado, tive ocasião de; entre outras observações, assistir a uma conferencia com demonstração pratica, do Prof. Hinsberg, chefe do Departamento de Quimica do Instituto de Patologia da Universidade de Berlim. A conferencia desse ilustre Prof. versou justamente sobre "Novos metodos para o diagnoticso do cancer". O que então disse o Prof. Hinsberg calou profundamente em meu espirito, tal a importancia de suas revelaçóes e por isso, ao findar o programa de excursões do Instituto, voltei a Berlim e procurei novamente aquele mestre para asenhorear-me de detalhes que nầo tinham sido peŕfeitamente entendidos durante a conferencia.

E', pois, sobre os trabalhos realizados pelo corpo de pesquizadores do Departamento chefiado pelo Prof. Hinsberg, em Berlim, que eu me proponho tratar nesta palestra, dando deste modo uma demonstração pratica da grande simpatia que me dispertơ a magnifica e oportuna obra ora encetata pela Liga de Combate ao Cancer do Centro Academico Oswaldo Cruz, á cuja primeira Diretoria, agora empossada, rendo minha homenagem augurando-lhe uma proficúa gestão.

O sangue e particularmente o sôro sanguineo tem sido de ha muito o substrato de um numero consideravel de reações para o diagnostico do cancer. Este fato demonstra desde logo que no espirito dos clinicos e pesquizadores se formou a conviçção de que o cancer, mau grado suas manifestações localizadas, não passa de uma expressão local de uma entidade morbida geral. Na realidade, variações qualitativas e quantitativas para o lado de diversos mponentes do sôro sanguineo têm sido constatadas em casos de cancẹr. E daí a insistencia com que os pesquizadores se esforçam por acharem uma reação quimica ou biologica caracterìstica para o diagnostico do cancer. Variações qualitativas e quantitativas nas frações albuminoides do sôro, no assucar, na fósfatase' na colesterina, etc., têm servido como reações indicadoras de lesões cancerosas. Quasi todas, entretanto, se revelaram inespecificas; dando resultados positivos no curso de varias enfermidades alem do cancer, especialmente a tuberculose, as lesões hepaticas, as inflamações e as doenças febris.

Hinsberg e seus colaboradores passaram em revista as mais importantes reações 'sorologicas até aqui conhecidas para o diagnostico do cancer, como as de Roffo, de Fuchs, de Bendien e de Botelho e concluiram pela inespecificidade de todas elas.

Sem entrar em detalhes sobre cada uma destas reações, acho todavia de interesse citar alguns dados relativos ás mesmas.

$$
\text { * * * }
$$

A reação de Roffo consta, em resumo, do seguinte: a 2 c.c. de sôro sanguineo obtido por simples caagulação do sangue sob parafina, 
juntam-se duas goas de uma solução a 1:1000 de vermelho neutro. No caso de uma reação positiva, aparecerá uma coloração bordeaux e, sendo negativa; uma cóloração alaranjada. Repetida esta reação com soros carcinomatosos e normais, constatou-se o seguinte: De 28 soros carcinomatosos, apenas 8 fóram positivos e de 12 soros normais, 2 deram resultado positivo. Para Albers que tomou a si o encargo de experimentar a reação de Roffo, o fator pessoal na apreciação da coloração da mixtúra é muito variavel, porque em alguns casos a coloŕação não se mostrà claramente definida. Este fato levou a modificar-se a reação de Roffo, introduzindo-se uma avaliação colorịmetrica quantitativa por meio de um fotometro graduado. Mesmo assim, a reação de Roffo não demonstrou uma especificidade capaz de recomendal-a como caracteristica do cancer. Em 52 casos de cancer apenas 35 reações foram positivas, sendo 17 negativas.

$\mathrm{A}$ influencia do $\mathrm{CO}_{2}$ presente no soro e do $\mathrm{pH}^{\circ}$ sobre o comportamento da reação de Roff́o tem sidọ posta em evidencia pelos pesquizadores.

Entre nós, Otto Bier experimentou ainda a reação de Roffo com soro sanguineo de diversos animais e chegou á seguinte conclusão: "a reação do vermelho neutro não é causada por nenhuma substancia especifica do soro de cáncerosos, visto que tambem se produz com o soro sanguineo de varios animais na ausencia de qualquer processo neoplasico".

Os seguintes autores, citados por Albers, obtiveram igualmente resultados que demonstram a inespecificidade desta reação.

\begin{tabular}{|c|c|c|}
\hline & $\begin{array}{c}\text { Reaçāo positiva em } \\
\text { casos de cancer } \\
\%\end{array}$ & $\begin{array}{c}\text { Reaçāo positiva em } \\
\text { casos de controle } \\
\%\end{array}$ \\
\hline Baje & 50 & 30 \\
\hline Hilarowisz & 30 & 70 \\
\hline Avila $\ldots \ldots \ldots \ldots \ldots$ & 60 & - \\
\hline Maislich $\quad \ldots \ldots \ldots \ldots$ & 36 & 87,5 \\
\hline Beric $\ldots \ldots \ldots \ldots \ldots$ & 48 & 29 \\
\hline
\end{tabular}

Estes e outros resultados parecem demonstrar, mau grado a convicção em contrario do autor da reação e outros pesquizadores, que a reação de Roffo não é caracteristica do cancer.

A reação de Fuchs foi estudada, experimentalmente no Departamento do Prof. Hinsberg por Albers, Merten e Mucke. Ela se baseia nas observações de Freunde, Kaminer e Neuberg "de que as celulas carcinomatosas são dissolvidas pelo soro normal, ao passo que as celulas normais o são pelo soro canceroso".

O mesmo fenomeno foi posteriormente constatato com a fibrina dos carcinomatosos e o soro normal e vice-versa. Para Fuchs, esta desintegração seria devida a um processo enzimatico semelhante ao 
que ocorre na reação antigeno-anticorpo. Acredita este autor ainda que a desintegração é de natureza especifica e como substituto da fibrina, usou ele proteinas de elevado peso molecular mais facilmente obtidas do que a fibrina.

$\mathrm{O}$ metodo de Fuchs foi repetido por varios investigadores e, tal qual acontece em outros casos, alguns se mostraram favoraveis, consignando mesmo uma percentagem de 92 a $94 \%$ de resultados corretos, ao passo que a mesma reação nas mãos de outros autores demonstrou uma inespecificidade digna de nota. Para alguns dos resultados falhos Fuchs, admite o uso de maus reagentes, explicando deste modo o fracasso de sua prova.

Albers, Merten e Mucke, auxiliados por dois dos colaboradores do proprio Fuchs, os doutores Höhler e Kreuscher, observaram o seguinte. Realizaram eles 400 exames em 247 soros. Os primeiros 104 casos foram desprezados, porque somente pouco a pouco puderam ser excluidas as causas de erro. Suas principais observações dizem respeito a 166 casos. De 94 soros cancerosos indubitaveis, apenas 30 deram resultado positivo, 29 foram negativos e 35 inativos. De 70 soros não cancerosos, para controle, 28 foram negativos, 16 positivos e 26 inativos. São as seguintes as conclusões dos autores: 1) 1/3 dos casos de carcinoma dá resultados falsos; 2) casos outros què nãa de cancer dão tambem resultados positivos; 3) um grande numero de soros é inativo, não dando resultado algum. Nãó admitem eles entâo a especificidade da reação de $\mathrm{Fuch}$, visto como nem o soró normal dá regularmente uma desintegração das celulas carcinomatosas, nem o soro carcinomatoso lisa sempre as celulas normaes.

Outras observações interessantes foram ainda feitas pelos autores no tocante á reação de Fuchs. Acreditam eles que a discrepancia constatada entre suas observações e as dos autores que se mostraram favoraveis a esta reação, reside no fato de que estes não excluiram de suas estatisticas os casos de cancer duvidơsos, bem como não desprezaram os casos de soros inativos. Tivessem eles tomado tais precauções, suas estatitisticas seriam bem diferentes.

Merten e Mucke realizaram áinda algumas verificações experimentais com a tecnica de Fuchs modificada por Minibeck, segundo a qual a dosagem èxata do azoto residual é substituida pela titulação dos amino-grupos. Tal modificação permite revelar diferenças de apenas 0,23 mg. por cento, nas analises. Esses autores experimentaram esta nova tecnica em 105 soros dos quais 46 eram de individuos sãos ou não cancerosos; 47 de cancerosos não tratados; 5 de cancerosos tratados, isto é,-irradiados; 3 de sarcomas e 4 de linfogranulomas. Dos 46 soros normais ou não cancerosos, 26 deram resultados corretos, 5 se mostraram inativos e 15 positivos. Dos 47 soros cancerosos, 40 deram resultados corretos, 5 inativos e 2 falsos. Nos casos irradiados, 1 foi positivo e outro negativo. Concluiram os autores: 1) a modificação da reação de Fuchs segundo Minibeck, por 
ser de realização mais clara, é preferivel á original è tem menos causas de erro; 2) só uma pequena percentagem de soros cancerosos dá reação falsa, mas uma grande percentagem de soros de processos inflamatorios dá igualmente reação falsa; 3) estes falsos resultados dependem principalmente da escolha do substrato. Finalmente, dizem eles, "estamos convencidos de que a modificação de Minibeck da reação de Fuchs para o cancer, com um bom substrato, possibilita um diagnostico de carcinoma, mas só em conjunto com outrà bôas reações".

A reação de Botelho parece ser a mais conhecida entre nós pelo interesse que dispertou em virtude da nacionalidade de seu autor. Ela se baseia na précipitåção dos coloides do soro sanguineo por meio de um. reativo iodọ-iodado em meio acido. A reaçấo acida do reagente é obtida com adição de uma mixtura de acido citrico e formalina. Posteriormente, para maior especifícidade da reação, o autor substituiu essa mixtura por uma solução a $1 \%$ de acido nitrico.

Merten foi o encarregado de verificar experimentalmente a eficacia da reação de Botelho.' Seus resultados foram, em, resumo, os seguintes: de 56 soros normais ou não cancerosos, 34 deram resultado negativo e 22 positivo; de 77 soros cancerosos (inclusive 7 sarcomatosos), 58 foram positivos e 15 negativos.

A influencia do teor em albumina dos soros foi particularmente estudada e de tudo o que Merten poude observar em suas experiencias, concluiu ele que a reação de Botelho, apezer de não ser especifica do cancer, dando frequentemente resultados positivos falsos em certas doenças como por exemplo, ulcus, diabetes, tuberculose e inflamações, pode ser aproveitada si praticada conjuntamente com outras reações para o cancer.

Merten estudou tambem uma outra reação, a de Bendien. Tal reação se baseia tambem na precipitação das substancias albuminoides do soro sanguineo por meio de um reagente composto de ortovanadato de sodio e acido acetico. Como nos demais casos, esta reação tem demonstrado bons e maus resultados nas mãos 'de outros pesquizadores que a praticaram. Merten observou o seguinte com a reação de Bendien: de 24 soros carcinomatosos, 4 deram resultado negativo (carcinomas do estomago, do recto e do bronquio); de 20 casos de controle, soros não carcinomatosos, 9 foram positivos (diabetes, ictęricia, colecistite, tuberculose e doenças febris).' Concluiu aquele autor pela ausencia de valor diagnostico em tal reação.

$* \quad * \quad *$

Albers, de um lado e Hinsberg e Merten, de outro, investigaram, respectivamente, as taxas de fosfatase e de assucar combinado em 
soros carcinomatosos e não carcinomatosos., Relativamente á fosfatase, observou-se que o teor deste elemento é, de um modo geral, baixo nas doenças, especialmente nos casos de processos ulcerativos do estomago e intestino e na tuberculose pulmonar.

Para a dosagem da fosfatase no soro sanguineo o autor usou o metódo de Jenner e Kay:

Hinsberg e Merten investigaram o valor da taxa de assucar combinado no soro sanguineo como possivel indicação para o diagnostico do cancer. A dosagem do assucar combinado foi feita pelor metodo francez de Bierry-Fandard com a modificação italiana de Condorelli. Os resultados obtidos pelos autores foram os seguintes. Em determinadas enfermidades, inclusive o cancer, a taxa do assucar combinado acha-se aumentada, como nas afecções do figado, na tuberculose e na leucemia. A dosagem do assucar combinado apresenta algum valor visto como, pelo exame clinico, podem ser excluidas as afeç̧ões que igualmente como o cancer são capazes de demonstrar altos valores de assucar combinado no soro sanguineo, não havendo, entretanto, nenhuma especificidade messa prova.

$$
* *, *
$$

São estas as reações quimicas ou fisico-quimicas experimentadáas no Departamento de Quimica do Instituto de Patologia sob a direção do Prof. Hinsberg. Quero agora referir uma outra serie de pesquizas ainda mais interessante no mesmo sentido, levada a efeito por uma assistente desse Departamento, a Doutora Guilhermina Rodewald, a quem se deve hoje, talvez, a mais especifica reação para o diagnostico do cancer.

$\mathrm{Na}$ conferencia pronunciada pelo Prof. Hinsberg sobre novos metodos para o diagnostico do cancer, a que aludi antes, disse esse cientista que tres eram os metodos por ele reputados como possuidores de especificidade bastante para que pudessem ser considerados como capazes de um diagnostico certo do cancer. Em primeiro lugar o metodo de Rodewald e depois os metodos polarografico e uma modificação da reação de Bendien proposta por Lowe, autor inglês.

Façamos primeiramente algumas rapidas referencias a estes dois ultimos para que encerremos esta palestra com os trabalhos de Rodewald.

O metodo polarográfico é praticado por meio de um aparelho chamado polarografo e se besaia no fato de que para a redução electrolitica de uma substancia é sempre necessaria uma determinada tensão eletrica, esta tensão sendo caracteristica de cada substancia. Quando a tensão electrolitica é baixa de mais, não haverá redução nem passagem de corrente. Por outro lado, a força da corrente eletrica necessaria á electrolise é diretamente proporcional' á concentração da substancia a ser reduzida. Este metodo polarografico presta-se para diversas investigações, servindo ás ánalises qualitativas e quantitativas nos-dominios da Quimica e da Fisico-Quimica, tendo já servido 
para a doșagem da albumina, da vitamina $\mathrm{C}$ e ainda ao soro diagnostico do cancer. Para o diagnostico do cancer registra-se a corrente eletrica capaz de transformar a cistina em cisteina sendo que "pela corrente eletrica necessaria a taĺ redução pode-se chegar ao diagnostico do cancer.

Para o Prof. Hinsberg a reação original de Bendien. só pode dar resultados qualitativos e daí a inespecificidade da mesma. A modificação, proposta por' Lowe, no entanto, aumenta consideravelmente a sensibilidade 'da reação, podendo se obter com ela resultados quantitativos bem nitidos. Em resumo, a modificação de Lơwe consiste no seguinte : cada soro a examinar é dividido em tres porções de 1c.c. A porçẫo A não é aquecida; a pórção B é inativada pelo calor e a porção $\mathrm{C}$ é agitada com eter. Do resultado obtido com a analisé combinada đessas tres porçôes poder-se-á chegar então ao diagnostico dò cancer. A quantidade do deposito obtido com a precipitação da albumina pelo reagente de Bendien, é avaliada por meio do interferometro de Lowe construido por Zeiss, de forma que resultados quantitativos muito mais seguros podem ser conseguidos por esta nova tecnica.

Vejamos agora os trabalhos de Rodewald. Esses trabalhos põem em clara evidencia as relações das glandulas endocrinas com o carcinoma, especialmente a hipofise. De um modo geral as pesquizas referentes ao papel da hiporise restringem-se á verificação da influencia que os hormonios hipofisarios podem exercer sobve o desenvolvimento dos tumores. Alterações histologicas têm sido postas em destaque nos casos de carcinoma: Voltam-se agora as vistas dos pesquizadores para as alterações funcionais dessa glandula. Verificou-se, por exemplo, que o teor em hormonio melanoforo ativo, das hipofises de individuós mortos por cancer é diminuido, em comparação com o observado èm outros casos de morte por molestias diferentes. Ao mesmo tempo o teor em hormonio melanoforo inativo acha-se aumentado nos casos de cancer, sendo que por meio da soda acustica este hormonio inativo podë ser ativado. Uma obșervação mais interessante é que nos animais com tumores implantados não se observam essas-alterações, as quais são, todavia, encontradas nos animais com tumores expontaneos. "Tratava-se, diz Rodewald, de animais de uma' linhagem na qual as femeas de 10 a 12 mezes eram afetadas de tumor. Nestas femeas apareciam os mesmos desvios hormonicos da hipofise presentes nos individuos com carcinoma".

Uma descoberta mais importante ainda é a de que no sangue dos cancerosos se encontra uma subștancia capaz de inativar o hormonio melanoforo da hipofise. Isto serviu de base para uma reação biologica aventada em 1937 e que, por deficiencia de tecnica, não deu os resultados esperados, demostrando. $20 \%$ de resultados falsos com soros humanos. Rodewald aperfeiçou a tecnica e conseguiu um novo metodo muito mais preciso, demonstrando apenas $6 \%$ de resultados 
falsos. O metodo se baseia na extração do hormonio melanoforo da hipofise posto então em contáto in vitro com o soro sanguineo a examinar. A mixtura é então injetada em rãs previamente colocadas' em condições de luz necessarias para o clareamento da pele. Sí o soro não é carcinomatoso, haverá um escurecimento da pele dâ rã por ação do hormonio sobre os melanoforos da mesma; si o soro é carcinomatoso, um antihơrmonio nele presente inativará o hormonio melanoforo e não haveră entấo mudança alguma na coloração da pele do animal:

Melhor, porem, do que esta reação baseada no poder do hormonio melanoforo é 'uma outra imaginada ainda por Rodewald e na qual o Prof. Hinsberg deposita a maior confiança. Trata-se da constatação de um antihormonio no soro sanguineo dos carcinomatosos capaz de inativar o hormonio gonadotropico da hipofise. $O$ animal de reação é a camondonga jovem sobre a qual o hormonio gonadotropico hipofisario provoca nitidas modificaçốes, para o lado dos orgãos genitais. Eu tive ocasião de assistir uma demonstração pratica desta reação e posso testemunhar a nitidez das modificações provocadas.

Neste caso não só o soro carcinomatoso possue um antihormonio gonadotropico, facilmente observavel com esta reaçâa biologica, como tambem o soro não carcinomatoso reforça a propriedade gonadotro-

\begin{tabular}{|c|c|c|c|c|}
\hline \multirow{2}{*}{ Molestia } & \multirow{2}{*}{ Idade } & \multirow{2}{*}{ Sexo } & \multicolumn{2}{|c|}{ Reação do hormonio } \\
\hline & & & Reforçada & Inhibida \\
\hline $\begin{array}{l}\text { Trombose venosa } \ldots \ldots \\
\text { Pielite } \ldots \ldots \ldots \ldots \ldots \\
\text { Fibrose da mama } \ldots \ldots \\
\text { Uremia } \ldots \ldots \ldots \ldots \ldots \\
\text { Meningite } \ldots \ldots \ldots \ldots \\
\text { Hipernefroma e Ictericia } \\
\text { Osteomalacia } \ldots . . . \ldots \ldots\end{array}$ & $\begin{array}{l}46 \\
\\
\\
66 \\
36 \\
57 \\
74\end{array}$ & $\begin{array}{c}\text { feminino } \\
\text { masculino } \\
\text { " } \\
" \\
"\end{array}$ & $\begin{array}{c}+ \\
++ \\
+ \\
++ \\
++ \\
+ \\
+\end{array}$ & \\
\hline 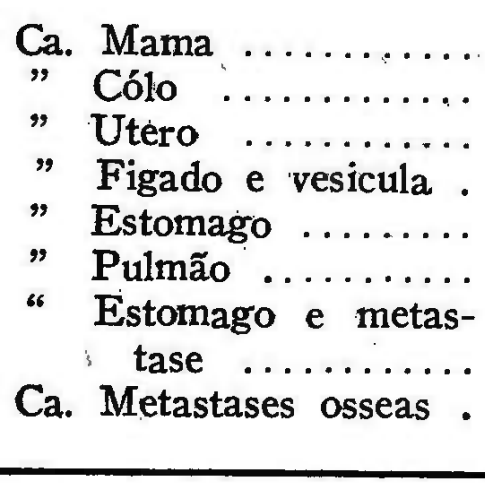 & $\begin{array}{l}36 \\
63 \\
58 \\
66 \\
56 \\
69 \\
55\end{array}$ & $\begin{array}{c}\text { feminino } \\
\text { " } \\
\text { masculino } \\
\text { " }\end{array}$ & & $\begin{array}{l}-\overline{(-)} \\
- \\
- \\
(-) \\
-\rightarrow \\
--\end{array}$ \\
\hline
\end{tabular}

pica do hormonio hipofisario, o que concorre sensivelmente para uma maior nitidez da reação. O quadro anterior exemplifica claramente os resultados obtidos por Rodewald com sua prova. 
Eitel, em 1938, constatou a presença no soro carcinomatoso de um antihormonio capaz de inativar o hormonio tireotropico da hipofise. Rodewald falando da ação antihormonal do soro carcinomatoso, é de opinião que "não se deve tratar de substancias distintas e especificas contra os varios hormonios, mas sim de uma ação antagonica contra um complexo hormonal, em geral".

Recorda Rodewald as observações de Sauerbruck e Knake sobre as perturbações na função sexual dos cancerosos, como tambem as que se passam no ciclo sexual dos animais com tendencia a tumores expontaneos e que "devem ser atribuidas a perturbações na produção do hormonio gonadotropico e que melhoraram com a administração de Prolan da hipofise". Demonstra isso que o hormonio gonadotropico tem uma ação favoravel sobre o tumor. Corroboram esta opinião as observações de Cramer e Horning que consiguiram evitar o aparecimento de carcinomas em uma linhagem de camondongos com tumores expontaneos, administrando aos mesmos um extrato hipofisario tireogonadotropico.

Pelo que vem de ser exposto, forçoso é concluir por uma eștreita relação entre as funcções hipofisarias e a formação dos tumores. As observações a que me referi têm, como disse no começo, uma capital importancia porque projectam inegavelmente muita luz sobre a sombra negra do cancer, atingindo os tres sectores em que se desdobra o problema dessa entidade morbida : o de sua etiologia, o de seu diagnostico e o de sua terapeutica.

Aos que por ventura se interessarem por estas questôes, terei a maxima satisfação em fornecer as indicações bibliograficas que possuo a respeito. 